\title{
Electrons in the supernova-driven interstellar medium
}

\section{Results from self-consistent time-dependent ionic and hydrodynamic evolution of the interstellar plasma}

\author{
Miguel A. de Avillez ${ }^{1,2}$, Gervásio J. Anela ${ }^{1}$, Ashish Asgekar ${ }^{3}$, \\ Dieter Breitschwerdt ${ }^{2}$, and Dominic H. F. M. Schnitzeler ${ }^{4}$
}

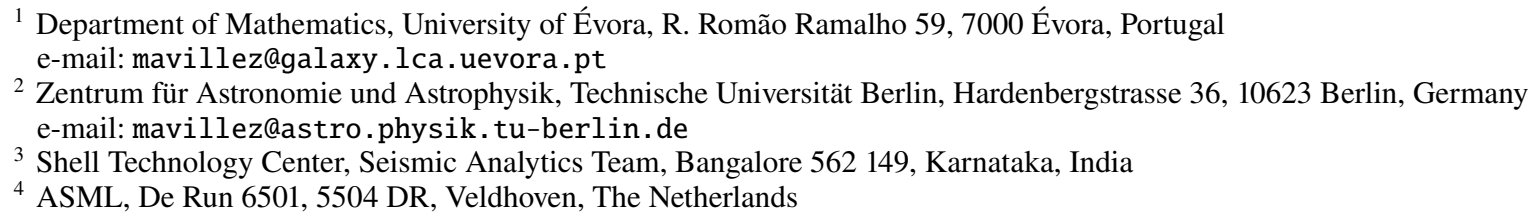

Received 31 July 2020 / Accepted 12 October 2020

\begin{abstract}
Context. Interstellar gas is in a highly turbulent dynamic state driven by successive supernova explosions and stellar winds, while its electron distribution is determined by microscopic processes such as ionization and recombination. In order to understand the properties of the electrons in the interstellar medium (ISM) it is necessary to follow numerically the nonlinear spatial and temporal evolution of the gas, its ionization structure, and its emission properties.

Aims. We study the time evolution of the electrons in the ISM and how line of sight observations compare to volume analysis of the simulated medium populated with atoms and ions of the ten most abundant species. In particular, we make quantitative predictions about the occupation fractions and averaged densities of electrons, the dispersion measures, and their vantage point dependence.

Methods. We carried out state-of-the-art adaptive mesh refinement simulations of the supernova-driven interstellar gas tracing the evolution of 112 ions and atoms of $\mathrm{H}, \mathrm{He}, \mathrm{C}, \mathrm{N}, \mathrm{O}, \mathrm{Ne}, \mathrm{Mg}, \mathrm{Si}, \mathrm{S}$, and $\mathrm{Fe}$ and their emissivities in a time-dependent fashion. The gas is followed with the magnetohydrodynamical adaptive mesh refinement parallel code coupled with the Collisional + Photo Ionization Plasma Emission Software to trace the ionic structure and radiative emission of the plasma.

Results. We show that more than $60 \%$ of the electrons are in thermally unstable regimes: about $50 \%$ at $200<T \leq 10^{3.9} \mathrm{~K}$ and $14 \%$ at $10^{4.2}<T \leq 10^{5.5} \mathrm{~K}$. The probability density functions for the electron distribution in different temperature regimes is rather broad, also a result of turbulence in the ISM. Comparing the calculated dispersion measures along different lines of sight to observation, we find a very good agreement. They increase linearly for distances greater than $300 \mathrm{pc}$ from the observer at an average rate of $27 \mathrm{~cm}^{-3}$ pc per kpc. The dispersion regarding the average dispersion measures does not decrease with distance along the line of sight, pointing to a high clumpiness of the electrons and of the turbulent ISM. The mean electron density in the Galactic midplane derived from the volume analysis varies between 0.029 and $0.031 \mathrm{~cm}^{-3}$, while that derived from the dispersion measures, varies between 0.0264 and $0.03 \mathrm{~cm}^{-3}$ depending on the vantage point and on the time averaged period. These variations can be as high as $8.3 \%$ between vantage points.
\end{abstract}

Key words. hydrodynamics - ISM: structure - Galaxy: disk - atomic processes - radiation mechanisms: thermal - plasmas

\section{Introduction}

The interstellar medium (ISM) is populated with free electrons resulting from collisional ionization and photoionization of atoms and ions, which can be followed with the ejection of Auger electrons as result of deep shell ionization (Pradhan \& Nahar 2011).

The current understanding of the distribution of free electrons in the ISM stems from observations, along the line of sight (LOS) towards pulsars with known distances, and from the adopted models for the electron distribution in the Galaxy (see, e.g., Cordes \& Lazio 2002, 2003; Yao et al. 2017; Schnitzeler 2012). The observations give the column density of the electrons (dispersion measure) denoted by DM $=\int_{0}^{L} n_{\mathrm{e}} \mathrm{d} l$, where $L$ is the length (pc) of the LOS and $n_{\mathrm{e}}$ is the electron density $\left(\mathrm{cm}^{-3}\right)$. Knowing DM it is possible to derive the mean electron density by $\left\langle n_{\mathrm{e}}\right\rangle=\mathrm{DM} / L$. Dispersion measures against pulsars indicate that the mean electron density in the Galactic plane varies between 0.02 and $0.1 \mathrm{~cm}^{-3}$ in the spiral arm, and between 0.01 and $0.17 \mathrm{~cm}^{-3}$ in the inter-arm region (see, e.g., Ferrière 2001; Gaensler et al. 2008; Berkhuijsen \& Fletcher 2008; Schnitzeler 2012; Yao et al. 2017).

Numerical simulations tracing the atoms and ions in the ISM in a time-dependent fashion, although scarce, are a valuable tool to understand, among other things, the time evolution of electrons and their distribution in the Galactic disk volume and along LOSs crossing the simulated volume, as well as their mean density at the Galactic midplane. The first non-equilibrium ionization simulations of the supernova-driven ISM published by de Avillez \& Breitschwerdt (2012a,b) and de Avillez et al. (2012) took into account electron impact and excitation-auto ionizations, and the ionization of HI by Lyman continuum photons 
resulting from helium recombination. However, although the interstellar radiation field was taken as a heating source, its effects on the ionic structure were not calculated.

Here we present results of new state-of-the-art adaptive mesh refinement simulations of the interstellar gas taking into account not only the photoionization due to the interstellar radiation field, but also a variable $\gamma$ (the ratio of the specific heats at constant pressure and volume) determined directly from the internal energy and whose value varies according to the ionization structure of the plasma with a maximum of $5 / 3$ (de Avillez et al. 2018).

The structure of the present paper is as follows. Sections 2 and 3 deal with a summary of the dynamical and thermal models of the ISM and the setup of the simulations, respectively. The overall evolution of the ISM (the time evolution of the electron density analyzed within the volume and along LOSs) is presented in Sect. 4. Section 5 closes the paper with a discussion and final remarks.

\section{Thermal and dynamical models}

The present simulations involve the joint dynamical and thermal evolution of interstellar gas in a self-consistent fashion with a direct coupling between the dynamics and the time-dependent evolution of the ionic structure of the plasma and its emission properties.

\subsection{Dynamical model}

The dynamical model is characterized by the following items: (i) a Eulerian description of the gas in a Cartesian coordinate system with $z$ representing the direction perpendicular to the Galactic midplane (located at $z=0 \mathrm{pc}$ in the $x-y$ plane); the total density $(\rho)$, the density of the 112 atoms and ions denoted by $\rho_{K, k}$ ( $K$ and $k$ are the atomic number and ionic state, respectively), the momentum $(\rho \boldsymbol{v})$, and total energy $(E)$ are determined by solving the Euler equations for the gas density, atoms and ions, momentum, and energy including the sources and sinks due to mass and energy ejection by supernovae, atom and ion emissivities, ionization, and recombination of the atoms and ions; (ii) the adiabatic parameter $\gamma$ is calculated on the fly from the gas internal energy following de Avillez et al. (2018); (iii) the equation of state $P_{\mathrm{th}}=(\gamma-1) \rho e-E_{\mathrm{si}}-E_{\mathrm{se}}$, where $e$ is the specific internal energy $(\rho e=E-\rho \boldsymbol{v} \cdot \boldsymbol{v} / 2)$, and $E_{\mathrm{si}}$ and $E_{\mathrm{se}}$ are the energies stored in ionization and excitation, respectively (de Avillez et al. 2018); (iv) a gravitational acceleration in the $z$-direction resulting from the contributions of the stellar disk (Kuijken \& Gilmore 1989) and a dark logarithmic halo (Helmi 2004); (v) heat conduction with saturated and classical components (Slavin \& Cox 1992) is included; (vi) an ultraviolet photon field is adopted, varying with the distance from the midplane (Wolfire et al. 1995) normalized in the Galactic midplane to the interstellar value near the Sun (Habing 1968); (vii) supernovae types Ia and Ib+c and II driving with the Galactic rates and scale heights described in de Avillez \& Breitschwerdt (2012b) is assumed.

\subsection{Thermal model}

The thermal model comprises the following: (i) interstellar gas composed of $\mathrm{H}, \mathrm{He}, \mathrm{C}, \mathrm{N}, \mathrm{O}, \mathrm{Ne}, \mathrm{Mg}, \mathrm{Si}, \mathrm{S}$, and $\mathrm{Fe}$ ions and atoms with solar abundances (Asplund et al. 2009, and the updates to the $\mathrm{Mg}, \mathrm{Al}, \mathrm{Si}, \mathrm{S}$, and $\mathrm{Fe}$ abundances by Scott et al. 2015b,a; see also Amarsi \& Asplund 2017); (ii) timedependent ionization structure of the plasma computed at each timestep; (iii) ionization due to electron impact and excitation auto-ionization, photoionization due to the UV photon field, charge exchange ionization with HII, HeII, and HeIII, and Auger and Coster-Kronig processes; (iv) secondary electrons that contribute not only to ionization, but also to excitation and deposition of energy as heat into the medium; (v) radiative and dielectronic recombination, charge-exchange recombination with HI, HeI, and HeII; (vi) radiative losses due to electron impact ionization, radiative and dielectronic recombinations, and line (allowed, semi-forbidden, and forbidden) and continuum (free-bound, two-photon, and free-free) emissions; (vii) level populations in each ion calculated assuming excitation or deexcitation equilibrium following de Avillez et al. (2018); and (viii) equal Maxwellian temperature for electrons and ions.

The photoionization cross sections are taken from Verner \& Yakovlev (1995) and Verner et al. (1996), while the chargeexchange model and data sources are presented in de Avillez $\&$ Breitschwerdt (2010). The remaining atomic data and the collisional-radiative model is detailed in de Avillez et al. (2018).

\subsection{Software}

The dynamical evolution is followed with a modified version of the magnetohydrodynamical adaptive mesh refinement parallel (MAP; Jiang et al. 2012) code coupled with the Collisional + Photo Ionization Plasma Emission Software (CPIPES; de Avillez 2018) to trace the ionic structure of the plasma. Ionization and recombination processes due to primary and secondary electrons are taken into account.

\section{Simulation description and setup}

We model over a period of 500 Myr a section of the Milky Way centered at the solar circle, having an area of $1 \mathrm{kpc}^{2}(0 \leq x, y \leq$ $1 \mathrm{kpc}$ ) and extending to $15 \mathrm{kpc}$ above and below the Galactic midplane $(-15 \leq z \leq 15 \mathrm{kpc})$ with the finest adaptive mesh refinement resolution of $0.5 \mathrm{pc}$ in the region $|z| \leq 1 \mathrm{kpc}$, corresponding to an effective grid cell number of $2000^{3}$ per $\mathrm{kpc}^{3}$. The coarse grid resolution is $4 \mathrm{pc}$. For $|z|>1 \mathrm{kpc}$ a single grid with a ratioed resolution, starting at $4 \mathrm{pc}$, was used in order to reduce the computational load. Periodic boundary conditions were adopted in the $x-z$ and $y-z$ faces and outflow boundary conditions following the prescription of Joung et al. (2012) were used at the top $(z=15 \mathrm{kpc})$ and bottom $(z=-15 \mathrm{kpc})$ boundaries $(x-y$ planes $)$

We are aware that such long evolution times result in a considerable shear of the grid due to differential Galactic rotation. In addition, the sound crossing time across the grid is much shorter than the evolution time, which makes the periodic boundary conditions prevalent. However, these conditions in neighboring computational boxes would be fairly similar. On the other hand, the small box size of $1 \mathrm{kpc}$ in the disk works against a large-scale shear, but more importantly the flow is governed by local energy and momentum sources, such as supernovae and stellar winds, and hence their resulting turbulence. Therefore, the aim in this paper is to focus on the feedback due to supernovae and stellar winds while studying the electron distribution.

\section{Electron distribution in the simulated disk}

Figure 1 displays the total electron density (in $\mathrm{cm}^{-3}$ ) distribution in the simulated Galactic midplane $(x-y$ plane at $z=0 \mathrm{pc})$ at 400 (top panel), 450 (middle panel), and 500 (bottom panel) 

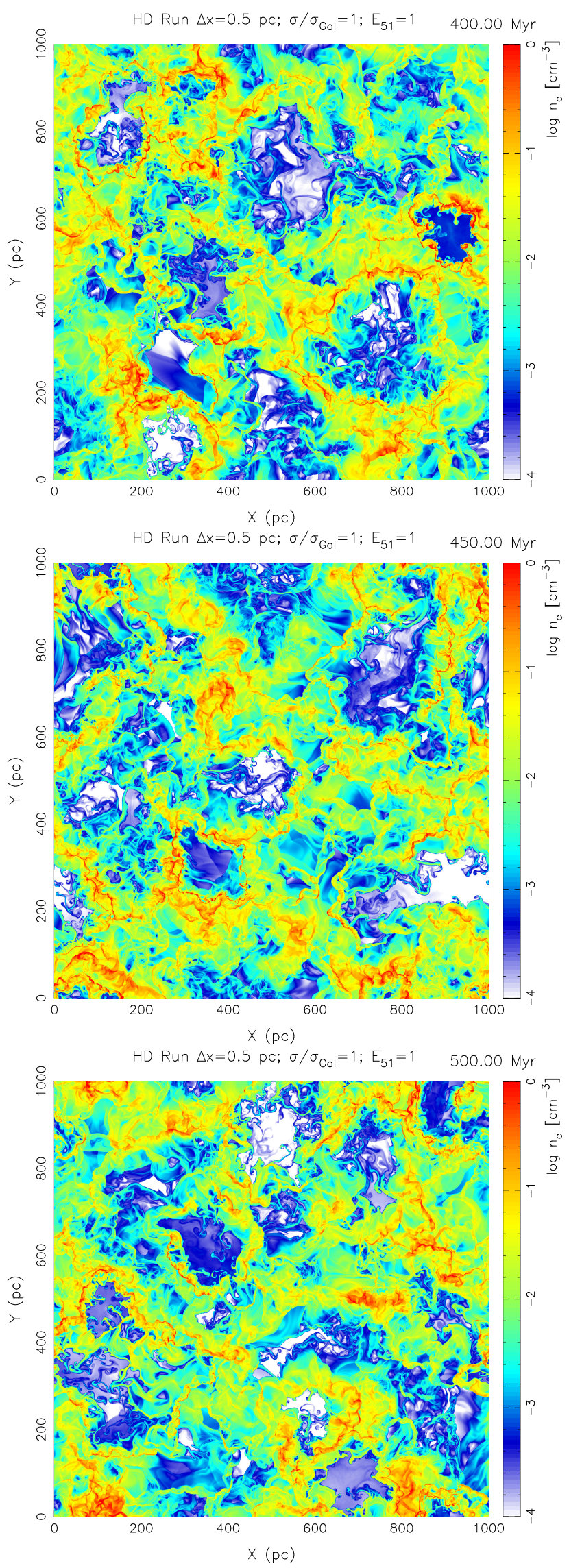

Fig. 1. Electron distribution (in $\log _{10}$ scale) in the simulated Galactic midplane at 400 (top), 450 (middle), and 500 (bottom) Myr of disk evolution.

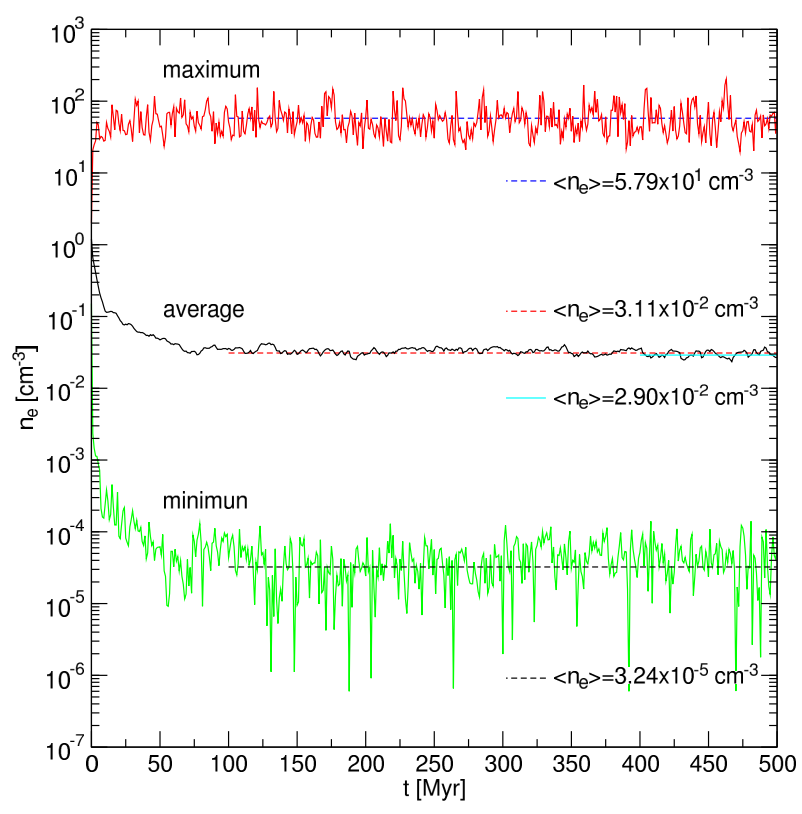

Fig. 2. Time evolution of the minimum (green), average (black), and maximum (red) electron density in the Galactic disk and time averages over 100-500 Myr (dashed black, red, and blue lines) and 400-500 Myr (cyan) of disk evolution are displayed.

Myr of disk evolution. In general the medium is composed of (i) low density cavities (also known as bubbles) surrounded by complete or broken shells; (ii) shock compressed gas between bubbles, often representing high density regions $\left(>10^{2} \mathrm{~cm}^{3}\right)$ that are formed as a result of the combined effects of shock waves and cooling, through radiative losses; (iii) filamentary structures resulting from the breakup of shells, which at initial times of evolution are spherical but soon change their geometry due to density gradients in the surrounding medium; (iv) filaments as a consequence of increasing vorticity and vortex tube stretching; (v) clumps distributed everywhere hosting electrons and ions with low and high ionic charges. Overall, the system has a frothy and diffuse appearance as a result of its clumpiness and the amount of small-scale structures.

Turbulent mixing layers are observed in the outer regions of the hot bubbles with material progressing from the shell into the bubble through finger-like structures (resulting from Rayleigh-Taylor and Kelvin-Helmholtz instabilities) that promote the transport of electrons and the mixing of the material (for a detailed study of turbulent mixing in the ISM see de Avillez \& Mac Low 2002). This evolution is a typical property of a turbulent medium driven by supernovae where the scales of the observed structures are proportional to some power of the Reynolds number of the flow, i.e., $l / \eta \propto \mathrm{Re}^{3 / 4}$ in incompressible turbulence (see, e.g., Davidson 2004; Elmegreen \& Scalo 2004). Here $l, \eta$, and Re are respectively the largest scale (injection scale) at which energy is injected into turbulence, the dissipation scale (the smallest scale at which energy is dissipated), and the Reynolds number, which is observed also in the compressible ISM simulations of de Avillez \& Breitschwerdt (2007).

\subsection{Volume analysis}

Over time the population of electrons in the disk varies due to the different ongoing atomic and dynamical (including turbulent) processes. Therefore, the time evolution of their minimum, 

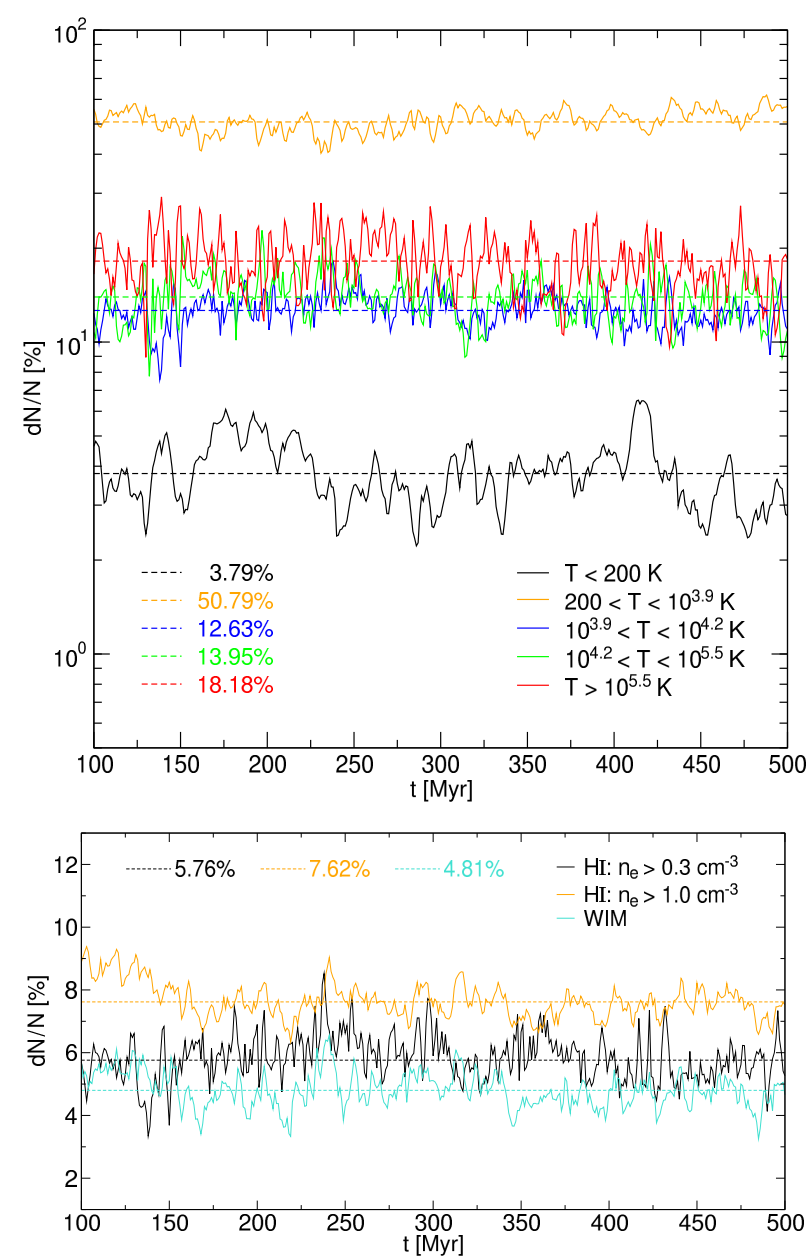

Fig. 3. Time evolution of the occupation fractions (percent of the disk volume) of the electrons associated with the different temperature regimes (top panel) and in $\mathrm{HI}$ regions and in the warm ionized medium (bottom panel).

maximum, and averaged density in each region of the Galactic disk is not constant and can have large oscillations, as shown in Fig. 2, which displays the evolution of the minimum, maximum, and average density of the electrons in the simulated Galactic midplane over the $500 \mathrm{Myr}$ of evolution. The time average of each plot is displayed with solid lines (for the average over the period 100-500 Myr). The time averages of the maximum is $57.9 \mathrm{~cm}^{-3}$, while the minimum time average is $3.24 \times 10^{-5} \mathrm{~cm}^{-3}$. The averaged density over the $100-500 \mathrm{Myr}$ period is $3.1 \times 10^{-2} \mathrm{~cm}^{-3}$ decreasing to $2.9 \times 10^{-2} \mathrm{~cm}^{-3}$ if the period 400-500 Myr is considered. We do not consider the first 100 Myr of disk evolution as it still reflects the initial conditions of the system (de Avillez \& Breitschwerdt 2004).

The time evolution (100-500 Myr) of the volume occupation fraction of the free electrons enclosed in different temperature regimes (stable: $T<200 \mathrm{~K}, 10^{3.9}<T \leq 10^{4.2} \mathrm{~K}$, and $T>$ $10^{5.5} \mathrm{~K}$; unstable: $200<T \leq 10^{3.9} \mathrm{~K}$ and $10^{4.2}<T \leq 10^{5.5} \mathrm{~K}$ ) are shown in the top panel of Fig. 3. The different regimes have time averaged values of $3.8 \%(T<200 \mathrm{~K}), 50.8 \%(200<$ $\left.T \leq 10^{3.9} \mathrm{~K}\right), 12.6 \%\left(10^{3.9}<T \leq 10^{4.2} \mathrm{~K}\right), 14 \%\left(10^{4.2}<T \leq\right.$ $\left.10^{5.5} \mathrm{~K}\right)$, and $18 \%$ for the hot gas $\left(T>10^{5.5} \mathrm{~K}\right)$. The fraction of volume occupied by electrons that are locked in HI regions with densities higher than 1 and $0.3 \mathrm{~cm}^{-3}$ have a time averaged value of 5.8 and $7.6 \%$, respectively, while electrons in the warm ionized medium (WIM, $6000<T \leq 10^{4} \mathrm{~K}$; Haffner et al. 2009)
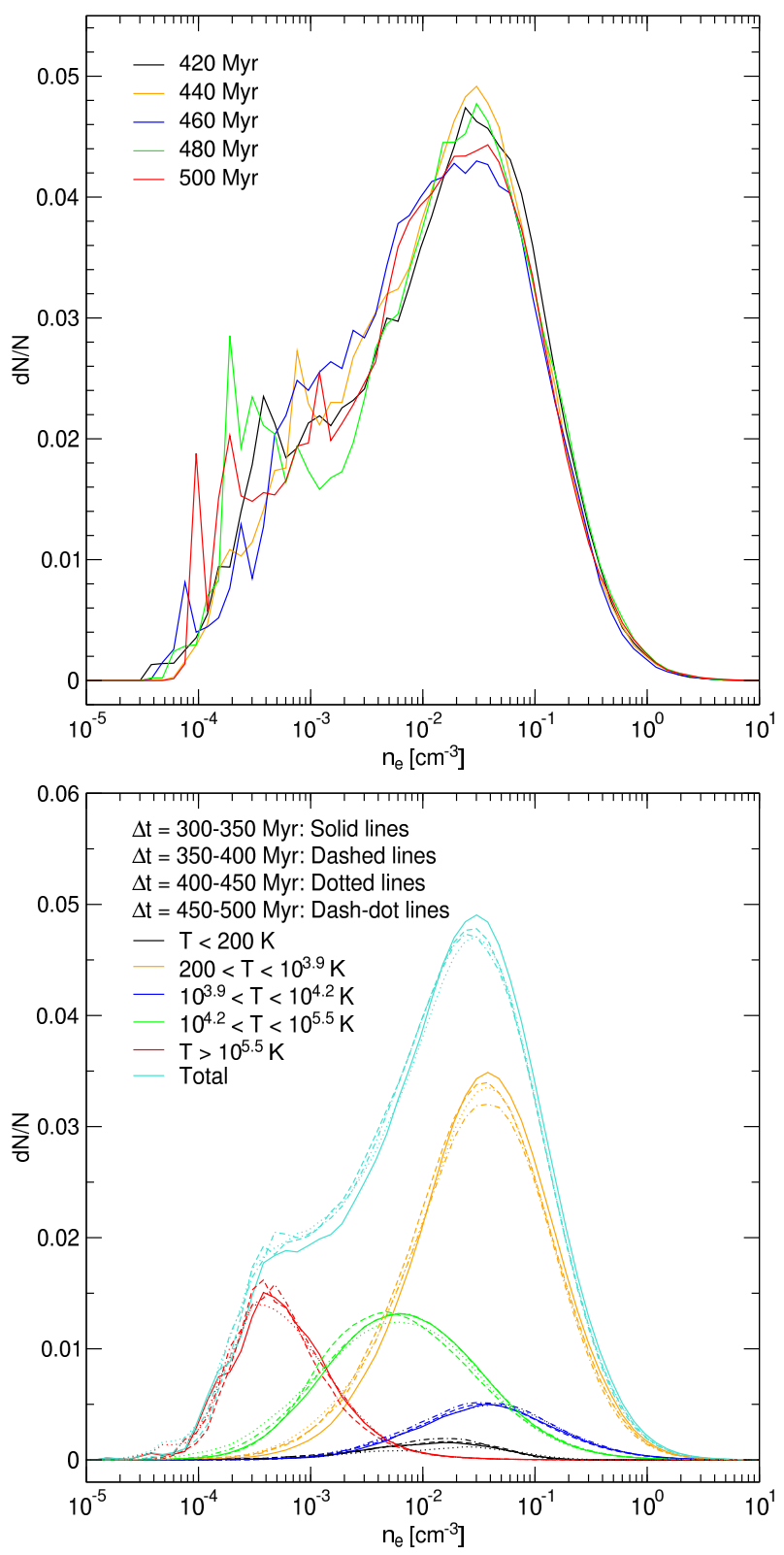

Fig. 4. Top panel: histograms of the electron density in the Galactic disk at different times from 400 through $500 \mathrm{Myr}$ at every $20 \mathrm{Myr}$. Bottom panel: time averaged volume histograms of the electron density in the Galactic disk for different temperature regimes and total density (brown) calculated between 400 and $500 \mathrm{Myr}$ using 51 snapshots with a time interval of $1 \mathrm{Myr}$.

occupy $4.8 \%$ of the simulated disk volume. These values are similar to those discussed in de Avillez et al. (2012) and are in line with the observed values (Berkhuijsen et al. 2006; Berkhuijsen \& Müller 2008; Gaensler et al. 2008). It is striking that more than $60 \%$ of the electrons are in classically thermally unstable regions.

Histograms of the electron distribution in the disk volume taken between 400 and 500 Myr with intervals of 20 Myr are shown in Fig. 4 (top panel). Although the histograms provide some information regarding the system at specific times, they are meaningful for the overall evolution of the free electrons in the ISM due to their variations, which are inherent to the underlying physical phenomena occurring at that time and captured in the corresponding histogram. These conditions may not repeat 
themselves at another time as the ISM is a nonlinear chaotic system. The spikes seen in the histograms are associated with the supernova activity, thus producing very hot gas (a few $10^{7} \mathrm{~K}$ ), in the disk leading to an increase in the number of electrons release by the different ions, most importantly from $\mathrm{Si}, \mathrm{S}$, and $\mathrm{Fe}$. Nevertheless, these histograms suggest the presence of electrons with a Gaussian distribution centered at $\simeq 3.0 \times 10^{-2} \mathrm{~cm}^{-3}$.

Moreover, we can see that the distribution of electrons (occupation fraction) becomes broader with time as successive supernova explosions drive the ISM, and hence electron density has more extreme values, i.e., denser regions become denser because of the compression of already compressed regions, and low density bubbles become less dense to due an increasing evacuation by each new explosion.

Even more information is provided by time averaged histograms (from $300 \mathrm{Myr}$ through $500 \mathrm{Myr}$, using snapshots at every 1 Myr for a total of 51 snapshots) of the free electron distribution in the simulated disk (bottom panel of Fig. 4) for different temperature regimes $\left(T \leq 200 \mathrm{~K}, 200<T \leq 10^{3.9} \mathrm{~K}\right.$, $10^{3.9}<T \leq 10^{4.2} \mathrm{~K}, 10^{4.2}<T \leq 10^{5.5} \mathrm{~K}$, and $\left.T>10^{5.5} \mathrm{~K}\right)$ and total density. Although there are several differences among the averaged histogram profiles, they show that the total distribution (cyan line) of electrons is broad with a pronounced peak at around $3 \times 10^{-2} \mathrm{~cm}^{-3}$ and are dominated by the thermally unstable gas at $200<T \leq 10^{3.9} \mathrm{~K}$ (totally unstable) centered at $4 \times 10^{-2} \mathrm{~cm}^{-3}$ and $10^{4.2}<T \leq 10^{5.5} \mathrm{~K}$ (partially unstable) for densities greater than $3 \times 10^{-3} \mathrm{~cm}^{-3}$. It also includes the contributions of the thermally stable regimes $(T \leq 200 \mathrm{~K}$ and $10^{3.9}<T \leq 10^{4.2} \mathrm{~K}$ ) at the level of few percent level. The cold gas below $200 \mathrm{~K}$ only harbors a very small fraction of electrons. At lower densities the total distribution is defined by the hot gas $\left(T>10^{5.5}\right)$ and by the thermally unstable gas with $10^{5.0}<T \leq$ $10^{5.5} \mathrm{~K}$. It is not surprising that the hot gas at $T>10^{5.5} \mathrm{~K}$, which has a large number of free electrons, contributes substantially to the total histogram at lower densities.

The time averaged histograms are fitted with a composition of two Gaussian distributions with mean values of $n_{\mathrm{e}}=4.1 \times$ $10^{-4} \mathrm{~cm}^{-3}$ determined by the hot gas and $n_{\mathrm{e}}=2.9 \times 10^{-2}-$ $3.1 \times 10^{-2} \mathrm{~cm}^{-3}$ for the right Gaussian distributions. The mean electron density is in line with the value measured in the simulated disk, as discussed above (see Fig. 2).

\subsection{Line of sight observations}

Using three vantage points $(\mathrm{A}, \mathrm{B}, \mathrm{C})$ in the simulated Galactic midplane located at A $(x=0, y=0, z=0) \mathrm{pc}, \mathrm{B}$ $(x=1000, y=0, z=0)$, and C $(x=1000, y=500, z=0)$, LOS measurements were taken at distances of up to $4000 \mathrm{pc}$ from the observer. The lines of sight span a projected area (onto the simulated Galactic midplane) with an angle of $80^{\circ}$ from locations A (ranging from $0^{\circ}$ to $80^{\circ}$; green lines) and $\mathrm{B}$ (ranging from $95^{\circ}$ to $175^{\circ}$; red lines) and $90^{\circ}$ from location $\mathrm{C}$ (ranging from $135^{\circ}$ to $225^{\circ}$; blue lines) at every $2^{\circ}$. Thus, a total of 41 (from locations $\mathrm{A}$ and $\mathrm{B}$ ) and 46 (from location $\mathrm{C}$ ) lines of sight were taken.

Figure 5 displays the vantage point locations and the projected areas onto the simulated Galactic midplane covered by the lines of sight taken from those locations and extending to $2 \mathrm{kpc}$. The measurements through distances longer than the linear side of the section of the simulated disk, shown in the figure by the square with black solid lines, takes advantage of the periodic boundary conditions adopted along the computational domain faces perpendicular to the Galactic midplane, thus adding up the data cubes as needed in the $x$ and $y$ directions, as shown in the figure by the dotted squares.

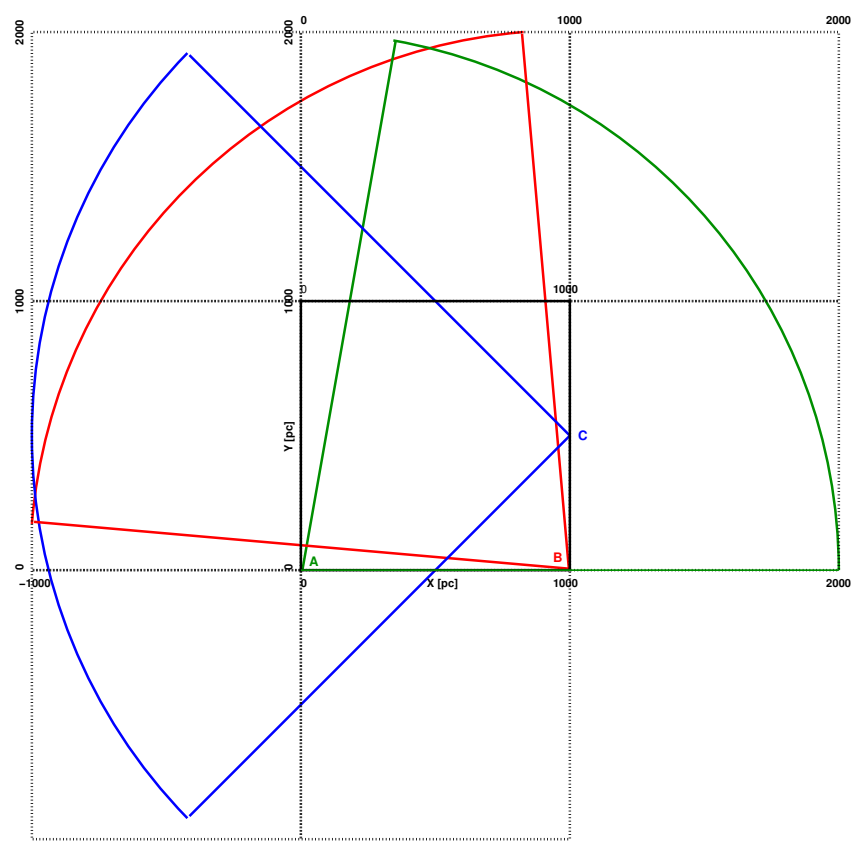

Fig. 5. Vantage points and areas projected onto the simulated Galactic midplane of $80^{\circ}$ and $90^{\circ}$ covered by the lines of sight taken from locations $\mathrm{A}, \mathrm{B}$, and $\mathrm{C}$ and extending over $2 \mathrm{kpc}$. The computational domain is represented by the solid black lines, while the replicated domains are represented by dotted lines.

\subsubsection{Dispersion measures}

The dispersion measures observed from the different vantage points vary between lines of sight and along lines of sight, as can be seen in Fig. 6, which displays the angular distribution of the electrons column density measured at 300 (top), 400 (middle), and 500 (bottom panel) Myr from the vantage points A (black lines), B (green lines), and C (red lines) for LOS with lengths 4000, 3000, 2000, 1000, 500, 100, and 10 (from top to bottom). For the sake of clarity the DMs from position $\mathrm{C}$ are deviated $25^{\circ}$ from their original angles $\left(135^{\circ}-225^{\circ}\right)$ in order to avoid overlap with the observations from position $\mathrm{B}$.

The LOSs trace the underlying topology of the interstellar gas as the lines cross regions with very different electron densities. Thus, regions with very low density (e.g., bubbles or supperbubbles) do not contribute much to the DM, and this is reflected in the dips seen in the angular distribution. Similarly, the presence of a shell or a cloud leads to an increase in the column density, hence a sudden increase in the DM, as can be seen in the figure. The largest variations in the dispersion measures at each position and between the different positions occur for $l_{\mathrm{LOS}}=10$ and $100 \mathrm{pc}$. The gap between the DMs at these distances varies between positions and in time (compare the two bottom lines for each position in each panel of Fig. 6). The dispersion measures for $l_{\mathrm{LOS}} \geq 500 \mathrm{pc}$ although varying over the angles, their positions in the different panels are similar between the three vantage points. We note that this distance is six times the correlation length $(75 \mathrm{pc})$ measured in the simulated ISM, thus a repetition pattern in the ISM occurs for a few correlation lengths (see, e.g., de Avillez \& Breitschwerdt 2007), which is imprinted in the spatial distribution of the DMs for larger LOS lengths.

Due to the variability of the topology of the turbulent ISM as a result of the ongoing processes the DMs observed at a specific time, because of their large variation among and along lines 


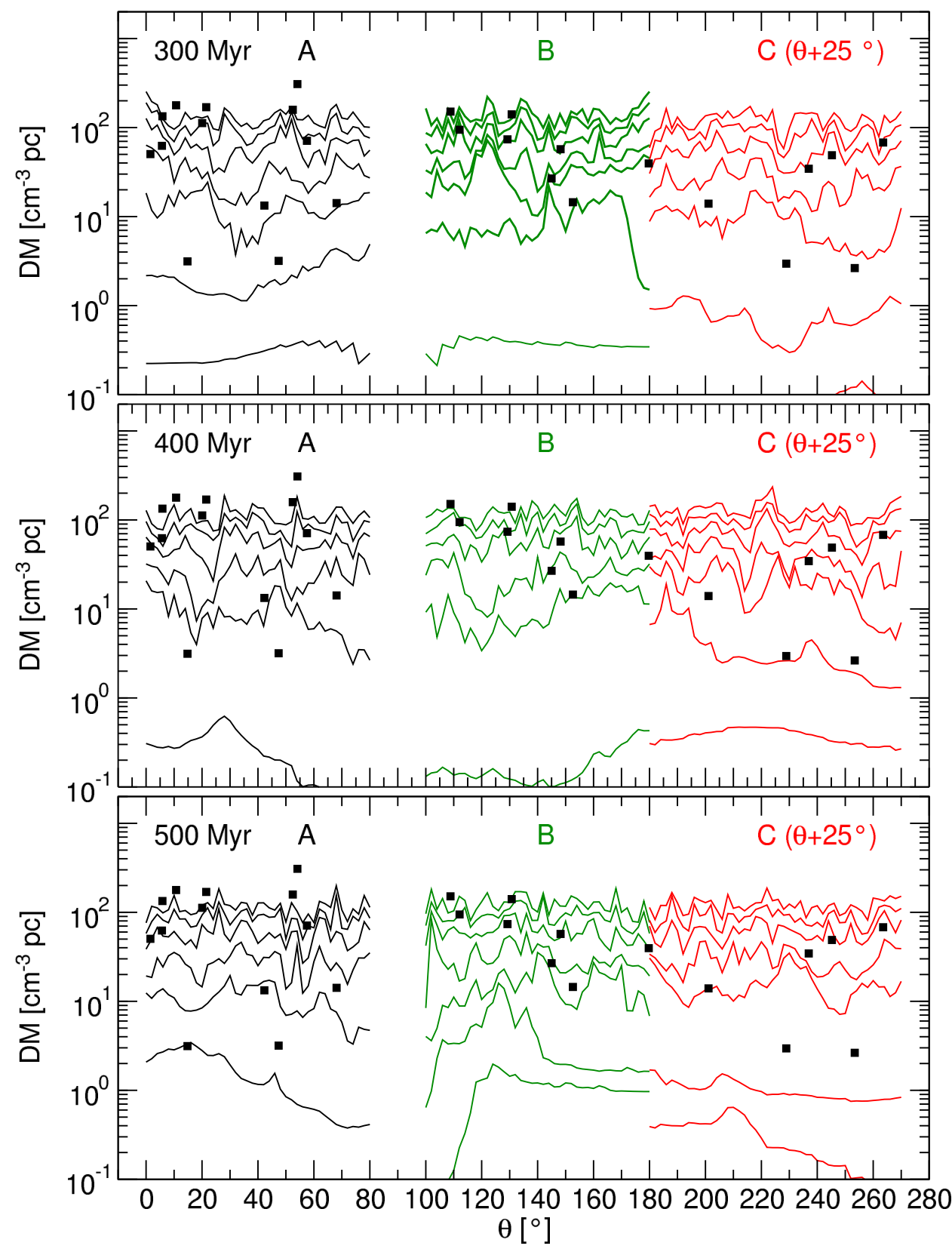

Fig. 6. Angular distribution of the dispersion measures observed from positions $\mathrm{A}$, $\mathrm{B}$, and $\mathrm{C}$ at (from top to bottom) 4000 , $3000,2000,1000,500,100$, and $10 \mathrm{pc}$. The data from position $\mathrm{C}$ is deviated by $25^{\circ}$ with regard to their original angles $\left(135^{\circ}-225^{\circ}\right)$ in order to avoid overlapping with the observations between positions B and $\mathrm{C}$. of sight, as can be seen in Fig. 6, are not representative of the system evolution overall. A more adequate indicator is the time average of the DMs combining different sets of data cubes spanning an evolution period of ISM. Figure 7 displays the minimum, average, and maximum of the time averaged DMs, $\langle\mathrm{DM}\rangle_{t}{ }^{1}$ $\left(\mathrm{cm}^{-3} \mathrm{pc}\right)$, determined up to $4 \mathrm{kpc}$ from the vantage points $\mathrm{A}$, $\mathrm{B}$, and $\mathrm{C}$ over 11,51 , and 101 data cubes with time intervals of $1 \mathrm{Myr}$ (in the ranges 300-310, 400-450 and 450-50, and 400-500 Myr). The figure also displays the dispersion measures obtained against pulsars of known distance located up to $200 \mathrm{pc}$ above and below the Galactic midplane and taken from de Avillez et al. (2012) and Yao et al. (2017).

After an initial steep rise in the vicinity of the vantage point (for LOS up to $300 \mathrm{pc}$ ) the dispersion measures show a linear growth with distance (with average values of 25-35 and $105-120 \mathrm{~cm}^{-3} \mathrm{pc}$ at 1000 and $4000 \mathrm{pc}$, respectively; see Table 1)

\footnotetext{
1 The average of the time averaged dispersion measure is denoted by $\left\langle\langle\mathrm{DM}\rangle_{t}\right\rangle$.
}

with an average increase of $27 \mathrm{~cm}^{-3} \mathrm{pc}$ per kpc in the DM. In addition, there is an almost constant dispersion (with distance) with regard to the average of $\langle\mathrm{DM}\rangle_{t}$ over 11, 51, and $101 \mathrm{Myr}$ averages.

With the increase in the number of data cubes used in the averaging process the smoothness in the curves increases (compare the evolution of the DM curves in all the plots) in particular for the LOS lengths smaller than $1 \mathrm{kpc}$. For larger LOSs the curves show a systematic smoothness. However, although the averaging process smooths out the larger variations on the electron distribution in the simulated ISM, the 50 and $100 \mathrm{Myr}$ averages still have the imprint of the underlying interstellar gas topology mostly resulting from long-lasting structures such as superbubbles that exist for tens of millions of years. Consequently, even with these longer averaging periods there is variability in the dispersion measures and in the mean electron density among the lines of sight and for the different vantage points. 
M. A. de Avillez et al.: Electrons in the supernova-driven interstellar medium
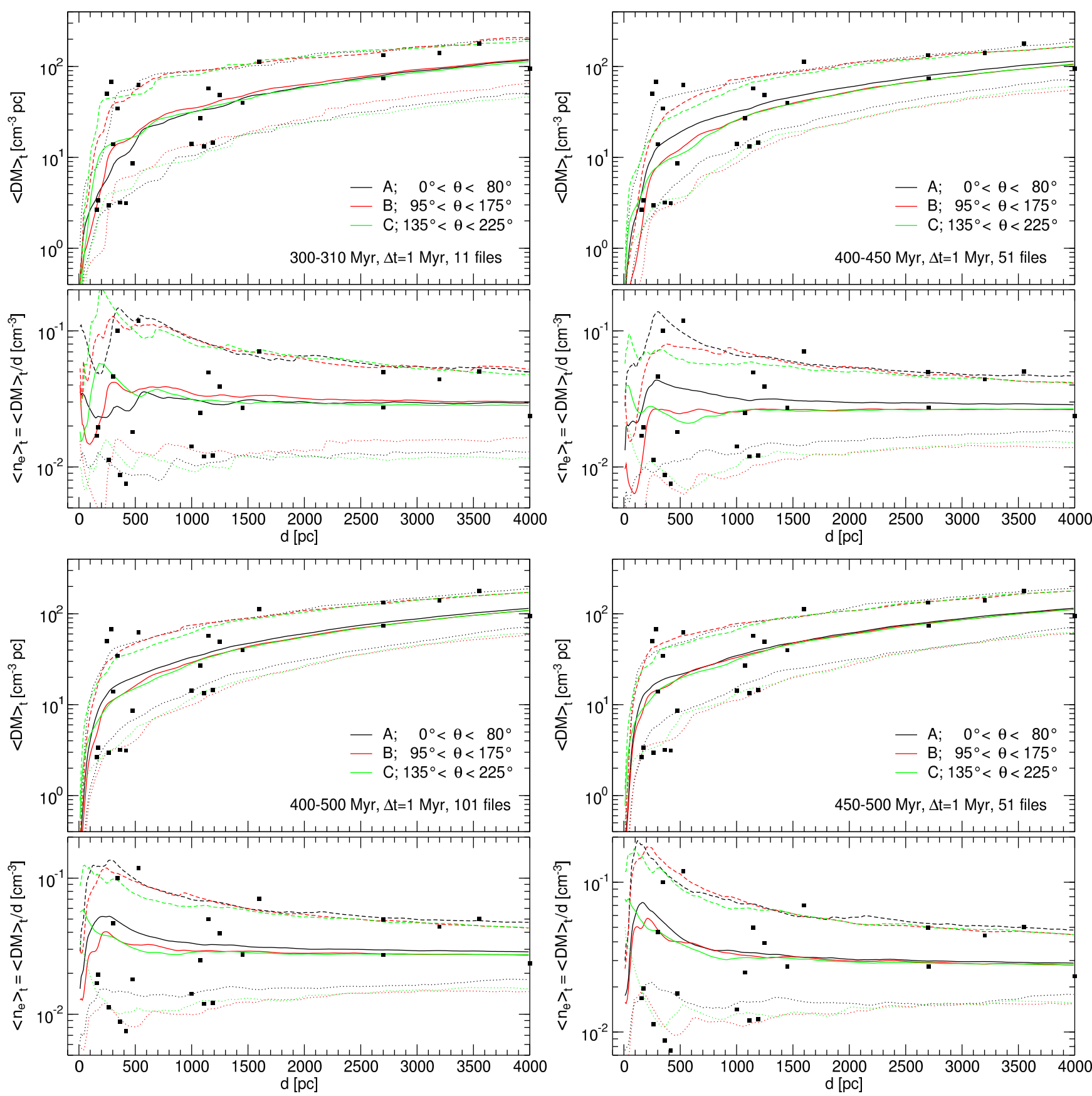

Fig. 7. Time averaged dispersion measures $\left(\langle\mathrm{DM}\rangle_{t}\right)$ and mean electron density $\left(\langle\mathrm{DM}\rangle_{t} / d\right)$ averaged over 11, 51, and $101 \mathrm{Myr}$ periods and measured from vantage points $\mathrm{A}, \mathrm{B}$, and $\mathrm{C}$. The black squares represent the $\mathrm{DM}$ and $\left\langle n_{\mathrm{e}}\right\rangle$ obtained against pulsars of known distance located at $|z| \leq 200 \mathrm{pc}$.

Overall the DMs, and the derived mean electron density, in the simulated disk are in line with the DMs obtained against pulsars of know distances with the maximum and minimum values of the DMs being in the interval defined by the observations.

The constancy of the time averaged dispersion measures with regard to the average shown in Fig. 7 is indicative of the spatial organization of the electrons in the ISM (see similar discussion, which we follow, in the context of Ovi observations in Bowen et al. 2008). If the LOSs intercept clouds of electrons with a fixed dispersion measure (e.g., $\mathrm{DM}_{\circ}$ ), then the total DM resulting from the interception of $N$ clouds would be $N \mathrm{DM}_{\circ}$ with a dispersion varying as $\pm \sqrt{N} \mathrm{DM}_{\circ}$. Hence, the dispersion should decrease with distance and approach the observed DM as the number of clouds increases and their DM decreases (Fig. 8). This reduction in the dispersion is not observed in Fig. 7. The observed clumpiness results from the fact that $\mathrm{SNe}$ increase the number and especially the density of shock compressed layers, and evacuate bubbles, so that the electron density becomes very inhomogeneous with time.

\subsubsection{Mean electron density}

The averaged observations from each vantage point in the simulated disk show a variability in the dispersion measures, and therefore, in the derived mean electron density; see bottom of each panel in Fig. 7, which displays the minimum, maximum, 
Table 1. Averages of the time averaged dispersion measures $\left(\left\langle\langle\mathrm{DM}\rangle_{t}\right\rangle\right)$ and derived mean electron density $\left.\left\langle\left\langle n_{\mathrm{e}}\right\rangle_{t}\right\rangle=\left\langle\langle\mathrm{DM}\rangle_{t} / d\right\rangle\right)$, calculated in 300-310, 400-450, 450-500, 400-500 Myr, for line of sight lengths of 1, 2, 3, and $4 \mathrm{kpc}$, and taken from the vantage points (VP) A, B, and $\mathrm{C}$.

\begin{tabular}{ccccccc}
\hline \hline VP & \multirow{2}{*}{$t[\mathrm{Myr}]$} & \multicolumn{4}{c}{$\left\langle\langle\mathrm{DM}\rangle_{t}\right\rangle\left[\mathrm{cm}^{-3} \mathrm{pc}\right]$} & $\left\langle\left\langle n_{\mathrm{e}}\right\rangle_{t}\right\rangle\left[10^{-2} \mathrm{~cm}^{-3}\right]$ \\
\cline { 3 - 6 } & & $1 \mathrm{kpc}$ & $2 \mathrm{kpc}$ & $3 \mathrm{kpc}$ & $4 \mathrm{kpc}$ & $4 \mathrm{kpc}$ \\
\hline A & $300-310$ & 31.37 & 60.17 & 89.23 & 117.50 & 2.95 \\
B & & 34.69 & 63.64 & 92.60 & 119.90 & 3.00 \\
C & & 31.38 & 58.96 & 86.02 & 112.80 & 2.83 \\
A & $400-500$ & 33.27 & 60.38 & 87.80 & 114.70 & 2.87 \\
B & & 29.41 & 56.09 & 82.63 & 108.50 & 2.72 \\
C & & 28.39 & 55.35 & 82.92 & 109.30 & 2.74 \\
A & $400-450$ & 31.94 & 59.56 & 87.33 & 114.30 & 2.86 \\
B & & 25.34 & 52.60 & 79.15 & 105.20 & 2.64 \\
C & & 25.70 & 51.83 & 79.92 & 106.70 & 2.67 \\
A & $450-500$ & 34.59 & 61.20 & 88.51 & 115.10 & 2.88 \\
B & & 32.99 & 59.58 & 86.12 & 111.80 & 2.80 \\
C & & 31.08 & 58.87 & 85.92 & 111.90 & 2.81 \\
\hline
\end{tabular}

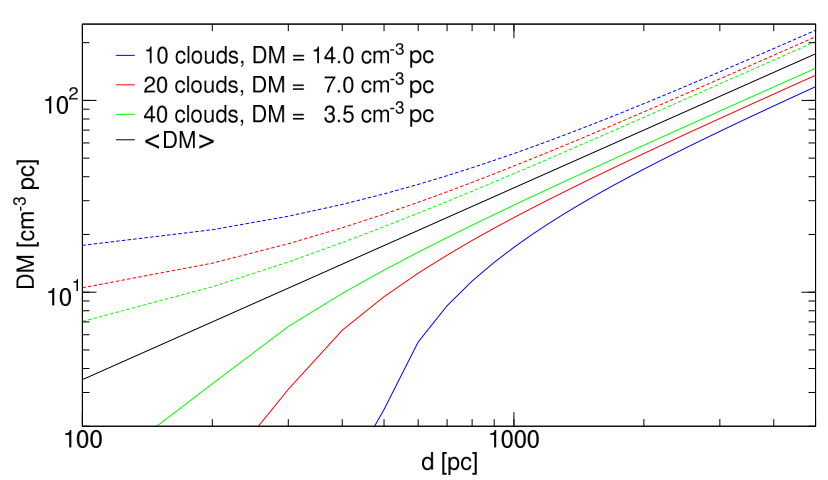

Fig. 8. Variation in the dispersion with distance as the LOSs cross 10, 20, and 40 clouds with DM of 14 (blue), 7 (red), and 3.5 (green) $\mathrm{cm}^{-3} \mathrm{pc}$.

and average electron density with distance derived from the time averaged dispersion measures (i.e., $\left.\left\langle n_{\mathrm{e}}\right\rangle_{t}=\langle\mathrm{DM}\rangle_{t} / d\left(\mathrm{~cm}^{-3}\right)\right)$. The average of the derived mean electron density is denoted $\left\langle\left\langle n_{\mathrm{e}}\right\rangle_{t}\right\rangle_{\mathrm{e}}$.

In the first $200 \mathrm{pc}$ from the vantage points the average $\left\langle n_{\mathrm{e}}\right\rangle_{t}$ increases to a maximum that varies among the different vantage points, the largest being $8 \times 10^{-2} \mathrm{~cm}^{-3}$ at position $\mathrm{C}$ for 450-500 Myr averages. After this initial rise in $\left\langle\left\langle n_{\mathrm{e}}\right\rangle_{t}\right\rangle$ there is a stabilization with distance towards the values measured for a LOS length of $4 \mathrm{kpc}$ and displayed in Table 1 . These densities not only vary between vantage points, but also according to the averaging period, and have a value between $2.64 \times 10^{-2}$ and $3 \times 10^{-2} \mathrm{~cm}^{-3}$, a variation of $13.6 \%$ with regard to the lowest value in the 7 th column of the table.

The averaging process did not provide a common value for the three vantage points other than smoothing their distance variations as the number of data cubes increases from 11 to 51 and 101. The electron density in the Galactic midplane varies between the vantage points for each averaging period, and can be as high as $6 \%$ for the $300-310 \mathrm{Myr}, 8.33 \%$ for the $400-450 \mathrm{Myr}$, $2.9 \%$ for the $450-500 \mathrm{Myr}$, and $5.5 \%$ for the $400-500 \mathrm{Myr}$ period average.

It should be pointed out that these values are consistent with the mean values derived from the averaged electron density PDFs obtained along the lines of sight and with the average electron density obtained through the volume analysis displayed in Fig. 2.

\section{Discussion and final remarks}

In this work we recalculated the electron distribution in the ISM using self-consistent three-dimensional time-dependent dynamical and ionic calculations of the interstellar gas composed of atoms, ions, and electrons due to the ten most abundant elements in nature. The calculations include, among other processes, charge transfer reactions, radiative and dielectronic recombination, electron impact ionization, excitation-auto ionization, and photoionization.

These calculations differ from the previous work of de Avillez et al. (2012) in the following ways: (i) inclusion of photoionization due to the interstellar radiation field (in addition to the other physical processes used in that work); (ii) inclusion of secondary electrons; (iii) calculation of the line emission using 30 levels per atom or ion, using up-to-date collision strengths (de Avillez et al. 2018); and (iv) update of rates associated with radiative and dielectronic recombination and charge-transfer. Therefore, the thermal evolution in the two simulations should show differences, for example in (1) the line emission (the level populations are better constrained than in the previous simulation where transitions to the ground state were assumed), (2) the emission due to the other processes as a result of updated rates, and (3) the ionic evolution of the plasma as a result of updated atomic data and the inclusion of photoionization. The last is responsible for the injection of photo-electrons, which in turn interact with other electrons and ions until their energy is degraded and deposited as heat in the medium (see, e.g., Shull 1979; Furlanetto \& Stoever 2010). The extent of these differences and how they affect the simulations, and in particular the electron distribution in the interstellar gas, is the subject of a forthcoming paper.

From the volume and LOS analysis it turns out that the mean electron density in the Galactic midplane depends on the methods used. The volume analysis shows a mean electron density of $\simeq 0.03 \mathrm{~cm}^{-3}$, while the LOS observations point to average 
values in the range $0.026-0.029 \mathrm{~cm}^{-3}$ and $0.027-0.029 \mathrm{~cm}^{-3}$ for averaged periods of 50 and $100 \mathrm{Myr}$, respectively. These values, although their differences are small, depend on the location of the vantage point.

The volume averaging includes the whole volume which tends to reduce the mean value, while the LOS average depends on the lines crossing the disk, and thus a fraction of the volume is missed. Hence, the averaged density obtained with the LOS can vary. Therefore, this selection effect should be taken into consideration when deriving the mean electron density. The results for the electron density in the Galactic midplane are in line with that obtained by Gaensler et al. (2008), Berkhuijsen \& Fletcher (2008), Schnitzeler (2012), and Yao et al. (2017), among others, and are $22.5 \%$ lower than the electron density in the Galactic midplane obtained by de Avillez et al. (2012).

As we found previously, the largest contributor to $n_{\mathrm{e}}$ comes from a thermally unstable phases of the ISM, with temperatures between 200 and $10^{3.9} \mathrm{~K}$ and between $10^{4.2}$ and $10^{5.5} \mathrm{~K}$, instead of the classical warm ISM with temperatures between $10^{3.9}$ and $10^{4.2} \mathrm{~K}$. The hot phase of the ISM has a very low free electron density of $4.1 \times 10^{-4} \mathrm{~cm}^{-3}$, while the combination of all other ISM phases has an average free electron density of about $0.03 \mathrm{~cm}^{-3}$.

Dispersion measures show large variations over time if sightlines are short $(\lesssim 100 \mathrm{pc})$. Over longer distances, the fluctuations become smaller through averaging. The ratio of the DM variation (of all points at a certain distance along the line of sight) to the average DM (of those points) does not decrease, which is caused by the many evacuated bubbles and shock compressed layers in the Galactic ISM. In a toy model where a line of sight intersects clouds of constant electron density, and where the number density of clouds is the same along the line of sight, the ratio of the DM fluctuations to DM decreases for longer lines of sight, which does not match the results of our simulations.

Acknowledgements. This research was supported by the projects "Hybrid computing using accelerators \& coprocessors - modelling nature with a novell approach" (PI: M.A.), InAlentejo program, CCDRA, Portugal, and "Enabling Green E-science for the SKA Research Infrastructure (ENGAGE SKA)", reference POCI-01-0145-FEDER-022217, funded by COMPETE 2020 and Foundation for Science and Technology (FCT), Portugal. The calculations were carried out at the Xeon Phi Orion cluster (Computational Astrophysics Group), while the data analytics was performed at the OBLIVION Supercomputer (ENGAGE SKA), both machines based at the University of Evora.

\section{References}

Amarsi, A. M., \& Asplund, M. 2017, MNRAS, 464, 264

Asplund, M., Grevesse, N., Sauval, A. J., \& Scott, P. 2009, ARA\&A, 47, 481 Berkhuijsen, E. M., \& Fletcher, A. 2008, MNRAS, 390, L19

Berkhuijsen, E. M., \& Müller, P. 2008, A\&A, 490, 179

Berkhuijsen, E. M., Mitra, D., \& Mueller, P. 2006, Astron. Nachr., 327, 82 Bowen, D. V., Jenkins, E. B., Tripp, T. M., et al. 2008, ApJS, 176, 59

Cordes, J. M., \& Lazio, T. J. W. 2002, ArXiv e-prints [astro-ph/0207156]

Cordes, J. M. \& Lazio, T. J. W. 2003, ArXiv e-prints [astro-ph/0301598]

Davidson, P. A. 2004, Turbulence: An Introduction for Scientists and Engineers (Orford: Oxford University Press)

de Avillez M. 2018, Cosmic Rays and the InterStellar Medium, 28

de Avillez, M. A., \& Breitschwerdt, D. 2004, A\&A, 425, 899

de Avillez, M. A., \& Breitschwerdt, D. 2007, ApJ, 665, L35

de Avillez, M. A. \& Breitschwerdt, D. 2010, ASP Conf. Ser., 438, 313

de Avillez, M. A., \& Breitschwerdt, D. 2012a, ApJ, 761, L19

de Avillez, M. A., \& Breitschwerdt, D. 2012b, ApJ, 756, L3

de Avillez, M. A., \& Mac Low M.-M. 2002, ApJ, 581, 1047

de Avillez, M. A., Asgekar, A., Breitschwerdt, D., \& Spitoni, E. 2012, MNRAS, 423, L107

de Avillez, M. A., Anela, G. J., \& Breitschwerdt, D. 2018, A\&A, 616, A58

Elmegreen, B. G., \& Scalo, J. 2004, ARA\&A, 42, 211

Ferrière, K. M. 2001, Rev. Mod. Phys., 73, 1031

Furlanetto, S. R., \& Stoever, S. J. 2010, MNRAS, 404, 1869

Gaensler, B. M., Madsen, G. J., Chatterjee, S., \& Mao, S. A. 2008, PASA, 25, 184

Habing, H. J. 1968, Bull. Astron. Inst. Netherlands, 19, 421

Haffner, L. M., Dettmar, R.-J., Beckman, J. E., et al. 2009, Rev. Mod. Phys., 81, 969

Helmi, A. 2004, MNRAS, 351, 643

Jiang, R. L., Fang, C., \& Chen, P. F. 2012, Comput. Phys. Commun., 183, 1617

Joung, M. R., Bryan, G. L., \& Putman, M. E. 2012, ApJ, 745, 148

Kuijken, K., \& Gilmore, G. 1989, MNRAS, 239, 651

Pradhan, A. K., \& Nahar, S. N. 2011, Atomic Astrophysics and Spectroscopy (Cambridge: Cambridge University Press)

Schnitzeler, D. H. F. M. 2012, MNRAS, 427, 664

Scott, P., Asplund, M., Grevesse, N., Bergemann, M., \& Sauval, A. J. 2015a, A\&A, 573, A26

Scott, P., Grevesse, N., Asplund, M., et al. 2015b, A\&A, 573, A25

Shull, J. M. 1979, ApJ, 234, 761

Slavin, J. D., \& Cox, D. P. 1992, ApJ, 392, 131

Verner, D. A., \& Yakovlev, D. G. 1995, A\&AS, 109, 125

Verner, D. A., Ferland, G. J., Korista, K. T., \& Yakovlev, D. G. 1996, ApJ, 465, 487

Wolfire, M. G., Hollenbach, D., McKee, C. F., Tielens, A. G. G. M., \& Bakes, E. L. O. 1995, ApJ, 443, 152

Yao, J. M., Manchester, R. N., \& Wang, N. 2017, ApJ, 835, 29 